\title{
Model for the static friction coefficient of spherical contact with a soft metal coating
}

\author{
Haibo Zhang ${ }^{1} \cdot$ Zhou Chen $^{1} \cdot$ Izhak Etsion ${ }^{1}$ (i)
}

Received: 18 March 2020 / Accepted: 30 May 2020 / Published online: 11 June 2020

(c) Springer Nature Switzerland AG 2020

\begin{abstract}
An elastic-plastic spherical contact with soft metallic coating under combined normal and tangential loading is studied by finite element analysis. Full-stick contact condition is assumed and sliding inception is related to vanishing tangential stiffness of the contact junction. Previously observed, both experimentally and theoretically, effects of increasing coating thickness on static friction coefficient that were published in the literature are thoroughly explained here, to the authors' best knowledge, for the first time. These effects include initial sharp drop of friction as soon as a thinnest coating film is applied, followed by a transitional behavior from decrease to increase of friction when the coating thickness is continuously increased. An intensive parametric study is performed and the effects of substrate and coating material properties on the static friction coefficient are revealed and thoroughly explained. An empirical expression for the static friction coefficient is derived along with the values for optimum coating thickness that provides the minimum friction coefficient.
\end{abstract}

Keywords Elastic-plastic contact $\cdot$ Spherical contact $\cdot$ Soft coating $\cdot$ Static friction

\section{List of symbols}

$a, b \quad$ Fitting parameters in the function of $\mu$

A Contact area

d Contact diameter

E Young's modulus

$K_{\mathrm{T}} \quad$ Tangential stiffness

$L_{c} \quad$ Critical load at yield inception in full stick contact condition

$P \quad$ Normal contact load

$P^{*} \quad$ Dimensionless normal contact load, $P^{*}=P / L_{c}$

$R \quad$ Radius of spherical substrate

$t \quad$ Coating thickness

$(t / R)_{\mathrm{m}}$ Dimensionless coating thickness for minimum friction

$u_{x} \quad$ Tangential displacement

$Y \quad$ Yield strength

$\mu \quad$ Static friction coefficient

$\mu_{\mathrm{m}} \quad$ Minimum static friction coefficient

$v \quad$ Poisson's ratio

$\omega \quad$ Interference

\section{Subscripts \\ co Coating material \\ su Substrate material \\ $0 \quad$ After normal loading \\ $\mathrm{s} \quad$ At sliding inception}

\section{Introduction}

To achieve and maintain higher efficiency and durability under increasingly severe contact conditions, protective coatings are becoming prevalent to control friction and wear in various kinds of contacts. These coatings can generally be divided into two broad categories [1]: "soft coating" and "hard coating". It is well known that the surface friction can be reduced by applying a soft coating with an appropriate thickness on the surface [2]. Owing to this friction reduction effect, soft coatings are usually used as solid lubricants especially in some industrial applications requiring contact without any type of liquid lubrication.

Izhak Etsion, etsion@technion.ac.il | 'Department of Mechanical Engineering, Technion, 32000 Haifa, Israel. 
The easily sheared soft metals, such as lead, indium, silver, and gold, are classical soft coatings [3]. Recently, addition of relatively softer $\mathrm{Cr}$ layer to hard diamond-like carbon (DLC) film showed reduction of friction under methane atmosphere [4]. However, trial and error procedure concerning both the choice of material and coating thickness is generally used to achieve good results. This is due to the lack of a general theoretical model that can satisfactorily describe the prevailing friction mechanisms in a tribological contact [5].

Bowden and Tabor [6] describe pioneering experiments on the friction coefficient of soft indium coated steel surfaces. They found that under a constant normal load, the friction coefficient first decreases and then increases as the coating thickness increases. Finkin [7] described this behavior of friction coefficient as related to ultrathin and thin films, respectively. In the ultrathin film regime, friction decreases with increasing film thickness, whereas in the thin film regime, friction increases with increasing film thickness.

The effect of coating thickness on friction was studied by other researchers with various explanations. For the ultrathin film regime, Finkin [8] explained, based on the stiffening of purely elastic thin layers in shear [9], that coated surfaces under shearing present an infinite friction coefficient when the film thickness approaches zero. This stiffening effect decreases with increasing coating thickness, leading to the decrease of friction.

For the thin film regime, Rabinowicz [10] assumed that the friction is affected by contributions from both the purely plastic soft coating and hard substrate. The increasing friction coefficient in this case is explained by decreasing mean contact pressure when coating thickness increases. Finkin [7] presented a purely elastic model for the effect of soft coating thickness on friction coefficient. Kato et al. [11] considered plastic deformation of the coating on a rigid substrate. Halling [12] considered purely elastic deformation for both coating and substrate, based on the numerical solution derived in [13] and plastic deformation based on empirical results from experiment. Chang [14] presented a model for a rough surface with an ion-plated soft metallic coating. It was assumed that the coating deformation is purely plastic while that of the substrate is elastic-plastic. However, a complete analysis of coated contact must consider possible elastic-plastic deformation for both the coating and the substrate. This was done by Gao et al. [15] who explained that the friction coefficient increases with increasing film thickness due to an increase in the contact area similar to [10].

To explain the observation in [6] for the two film thickness regimes, Liu et al. $[16,17]$ extended Chang's approach [14] by considering the DMT adhesion model [18]. They found a similar friction behavior as in [6] for a coated surface with low substrate roughness and under relatively low normal load.

In the aforementioned friction modeling work $[6,7$, 9-15], a coated surface was indented by a hard indenter. This may result in severe abrasive friction and wear due to plowing [19]. A good tribological design should avoid such indentation and hence, in the present study flattening, instead of indentation, of coated spherical contact that causes mild adhesive friction and wear is preferred.

The pioneering studies on contact behavior of a sphere under combined normal and tangential loading were conducted by Mindlin [20] in 1949 and Mindlin and Deresiewicz in 1953 [21] (an earlier paper in Italian is from 1938 by Cattaneo [22]). According to the purely elastic analytical solution of Mindlin, by applying a local Coulomb friction law, sliding inception always takes place at the contact interface. This contradicts the adhesive friction with material transfer when slip occurs below the contact interface [23]. A different method that considers sliding inception as a failure mechanism associated with plastic yield at the contact interface, was suggested by Bowden and Tabor [6], where the static friction coefficient is the material shear strength divided by the material hardness. This solution represents an extreme case of fully plastic contact interface, compared with the purely elastic contact in Mindlin's solution [20].

The complicated nature of frictional contact problems, such as elastic-plastic material behavior and boundary conditions at the contact interface, makes it difficult to acquire analytical solutions. For this reason, Brizmer et al. [24] developed a finite element (FE) model for elastic-plastic spherical contact subjected to combined normal and tangential loading. They assumed full-stick contact condition, which was validated experimentally in [25]. Sliding inception was related to vanishing tangential stiffness of the contact junction, associated with some failure below the contact interface inside the material. This assumption allows the well-known phenomenon of material transfer, which requires slip below the contact interface. The location where this slip occurs and the potential adhesive wear particle at sliding inception were studied in [26] based on the model in [24]. A different approach for the creation of adhesive wear particle, based on atomistic simulation [27] claims that a second mechanism with fracture at the bottom of the spherical asperity can also exist. However, the theoretical results of static friction coefficient in [24], which are based on plastic deformation, were validated experimentally in [28]. A detailed review of elastic plastic spherical contact under combined normal and tangential loading can be found in Ref. [29].

Chen and Etsion [30] extended the model in [24] to include hard coatings. They found that under a fixed normal load the static friction coefficient first increases 
then decreases with the increasing coating thickness. It was speculated in [30] that a sphere with soft coating will exhibit a mirror image behavior of a hard coating. This speculation was verified recently in [31] for a single combination of substrate and soft coating materials.

In the present paper, we extend the study in [31] to a larger range of material combinations and present a detailed explanation of the static friction behavior in soft metal coated spherical contacts. For simplicity, a full-stick contact condition is assumed and the material properties of the soft coating are assumed to be scale independent. The effects of dimensionless coating thickness are explained and those of material properties are revealed and explained by studying a wide range of these parameters. An empirical expression is derived for the static friction coefficient along with the value for optimum coating thickness that provides the lowest friction.

\section{Theoretical background}

Brizmer et al. [24] were the first to study full-stick contact between a homogeneous sphere and a rigid flat under normal and tangential loading, as shown in Fig. 1 (but without the coating). The material of the sphere was assumed elastic-plastic with a linear isotropic hardening. Initially, a load-controlled normal loading $P$ is applied on the rigid flat. This creates a contact area between the sphere and the flat. Then a displacement-controlled tangential loading $\left(u_{x}\right)_{i}$ increasing step by step ( $i$ is the step number) is applied. The increasing $u_{x}$ leads to an increasing tangential force $Q$. The tangential force $Q_{i}$ at the $i$ th step can be obtained by integrating the reaction forces along $x$-direction at the bottom ( $x y$ plane in Fig. 1). Thus, at each step the tangential stiffness of the contact junction $\left(K_{\mathrm{T}}\right)_{i}$ was calculated as

$\left(K_{\mathrm{T}}\right)_{i}=\left(\frac{\partial Q}{\partial u_{x}}\right)_{i} \approx \frac{Q_{i}-Q_{i-1}}{\left(u_{x}\right)_{i}-\left(u_{x}\right)_{i-1}}$

Because of the accumulated plasticity in the contact junction, $\left(K_{\mathrm{T}}\right)_{i}$ decreases during the tangential loading process, and sliding inception occurs when it vanishes. At this stage the maximum tangential force $Q_{\max }$ is reached, and the static friction coefficient $\mu$ is $Q_{\max } / P$. However, reaching this stage has a very high cost of computing time because the tangential stiffness decreases very slowly prior to vanishing. Thus, a drop to $10 \%$ of the initial tangential stiffness, i.e., $\left(K_{\mathrm{T}}\right)_{i} \leq 0.1\left(K_{\mathrm{T}}\right)_{1}$, was used in [24] as a criterion for sliding inception. Requiring lower tangential stiffness values negligibly increased the static friction coefficient $\mu$ by about $3 \%$ but dramatically increased the computation time [24, 31]. The initial tangential stiffness $\left(K_{\mathrm{T}}\right)_{1}$ is obtained by $Q_{1} /$
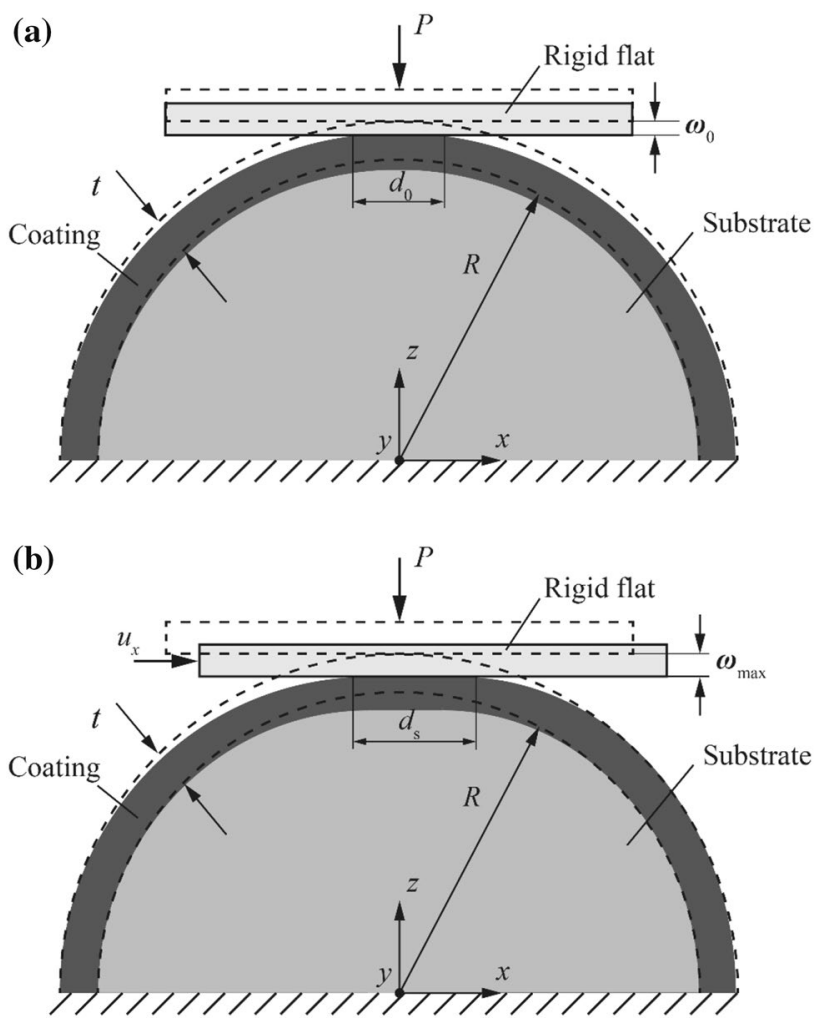

Fig. 1 Schematic of the contact between a sphere with coating and a rigid flat, under a normal load $P(\mathbf{a})$ and a subsequent tangential displacement $u_{x}(\mathbf{b})$

$\left(u_{x}\right)_{1}$. A sufficiently small $\left(u_{x}\right)_{1}$ is used to get an accurate initial tangential stiffness. The theoretical results of $\mu$ obtained in [24] with the above criterion for sliding inception were validated experimentally in [28].

By an extensive parametric study, Brizmer et al. [24] presented an empirical expression for $\mu$ as a function of dimensionless normal load as follows:

$\mu=0.27 \operatorname{coth}\left(0.27\left(P / L_{c}\right)^{0.35}\right)$

where $L_{c}$ is the critical normal load at yield inception of the sphere under full stick contact condition given by:

$L_{c}=\bar{L}_{c} \frac{\pi^{3} Y}{6} C_{v}^{3}\left(R\left(1-v^{2}\right) \frac{Y}{E}\right)^{2}$

where $\bar{L}_{c}=\left(8.88 v-10.13\left(v^{2}+0.089\right)\right)$ and $C_{v}=1.234+$ $1.256 \mathrm{v}$. The variables $R, v, E$, and $Y$ are the radius, Poisson's ratio, Young's modulus and yield strength of the sphere, respectively.

Based on [24] for homogeneous spherical contact, Chen and Etsion [30] studied the static friction coefficient of a spherical contact with hard coating. The model included a coating of thickness $t$ on a spherical substrate of radius $R$, with $Y_{\mathrm{co}} / Y_{\mathrm{su}}>1$ for hard coating case, where 
the subscripts "co" and "su" represent the coating and substrate, respectively. It was found that with increasing dimensionless coating thickness $t / R$, the static friction coefficient $\mu$ first increases then decreases having a maximum $\mu_{\mathrm{m}}$ at $t / R=(t / R)_{\mathrm{m}}$. For extremely thin or thick coating $\mu$ will approach $\mu_{\mathrm{su}}$ or $\mu_{\mathrm{co}}$, respectively, where $\mu_{\mathrm{su}}$ and $\mu_{\mathrm{co}}$ are the $\mu$ in a homogeneous spherical contact when the sphere is made of substrate or coating material, respectively, and can be obtained from Eq. (2). This is expected because in such cases the sphere with coating would behave like a homogeneous sphere.

Based on [30] for hard coating, Chen [31] studied the static friction coefficient of spherical contact including soft coating $\left(Y_{\mathrm{co}} / Y_{\mathrm{su}}<1\right)$ for a single combination of substrate and soft coating materials. It was found, see Fig. 2 (taken from Ref. [31]), that with increasing $t / R, \mu$ first decreases and then increases having a minimum $\mu_{\mathrm{m}}$ at $(t / R)_{\mathrm{m}}$. It was also noticed in [31] that as $t / R$ approaches zero the static friction coefficient does not approach $\mu_{\text {sur }}$ which differs from the observation in hard coatings [30]. The effect of normal loading on $\mu$ in the single case studied in Ref. [31] was also presented there, showing a decreasing $\mu$ with increasing normal load. However, neither detailed explanations for the coating thickness effects on static friction, nor the effects of various material properties were provided in Ref. [31].

\section{Finite element model}

In the present paper, the elastic-plastic coated spherical contact shown in Fig. 1 was studied for the case of soft metal coatings by a finite element (FE) model which was developed for hard coatings and verified in Ref. [30]. The

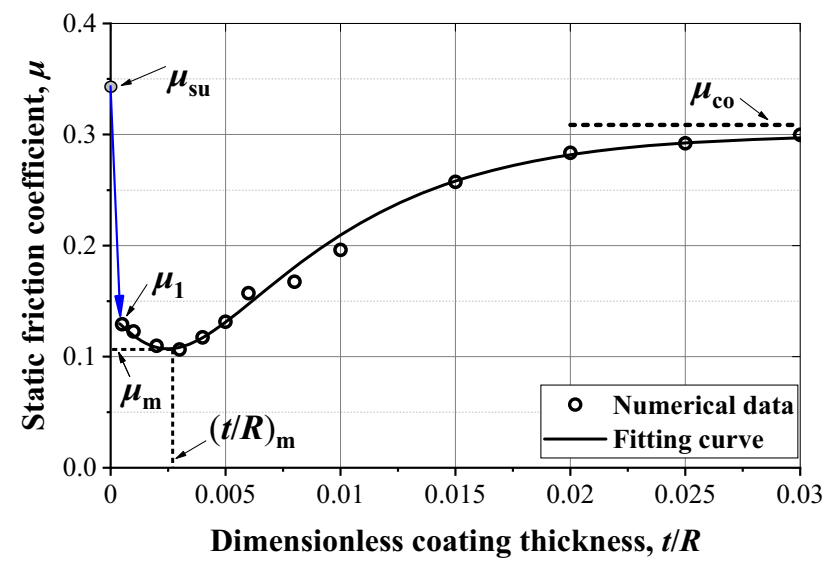

Fig. 2 The effect of the dimensionless soft coating thickness $t / R$ on the static friction coefficient $\mu$ with for a single combination $E_{\mathrm{co}} / E_{\mathrm{su}}=0.5, E_{\mathrm{co}} / Y_{\mathrm{co}}=1000, E_{\mathrm{su}} / Y_{\mathrm{su}}=1000$ and $P / L_{\mathrm{c} \text { co }}=100$ (taken from [31]). The numerical data are fitted by B-spline curve model is described in Ref. [30], hence only a brief summary is given in the following. The FE model is created in the software ANSYS 19.0. With symmetry condition on the plane $y=0$ as shown in Fig. 3, only a quarter of a coated sphere is used. The bottom (plane $z=0$ ) is fixed by constraining all the freedom of the nodes and perfect bonding is assumed at the coating/substrate interface. The full stick contact condition is applied at the contact interface, during both normal and tangential loading process. This means that for the newly created contact under tangential loading, further relative displacement of points engaged in contact is prevented (see Ref. [32]) and the lateral distance between any two points within the contact area cannot change (see Ref. [25]). This allows a potential material detachment in bulk, i.e., somewhere in the soft coating or substrate depending on the contact conditions.

Near the contact region the mesh is separated into three zones with different element size (see Fig. $3 b$ ). The smallest size is in Zone I, and the size increases with increasing distance from the contact interface. For more detailed description of the mesh in the different zones the reader is referred to Ref. [30].

To simplify the complex problem at hand and reduce the computation cost, the materials of coating and substrate are both assumed elastic-plastic isotropic based on classical continuum plasticity theory. The von Mises yielding criterion is used to determine the transition from elastic to plastic deformation (see, e.g., [30]). Hooke's and the Prandtl-Reuss constitutive laws governs the stress-strain relations in the elastic and plastic zones, respectively. Since the contact radius, $d_{s} / 2$ (see Fig. 1 ), is very small compared to sphere radius $R$, the analysis is carried out assuming

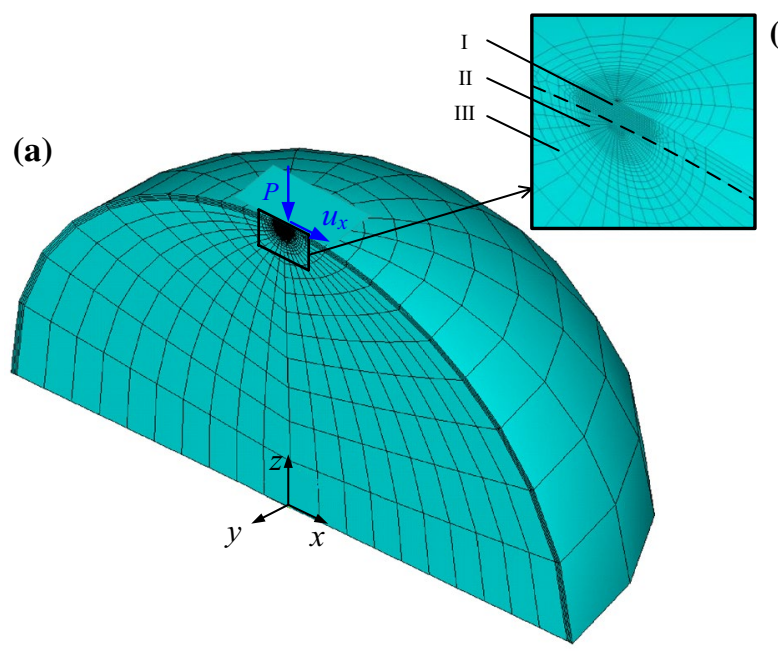

Fig. 3 Finite element model of a sphere with soft coating under combined normal and tangential loading (a) and three density mesh zones (b) 
small strain theory. A power-law hardening is adopted and the relation between the strain $\varepsilon$ and stress $\sigma$ is:

$\varepsilon= \begin{cases}\sigma / E, & \sigma<Y \\ (Y / E)(\sigma / Y)^{1 / n}, & \sigma \geq Y\end{cases}$

where $n$ is the strain hardening exponent. In the present study, we used $n=0.01$, which is typical for metals with small hardening [30]. Using a small hardening, improves the convergence of the FE model significantly compared to a case of elastic perfectly plastic material [24]. A Poisson's ratio $v=0.32$ is assumed for both the coating and substrate materials. Finally, for simplicity, the material properties of the soft coating are assumed scale independent. According to Ref. [33], the yield strength of small size samples is higher than the typical value of bulk due to dislocation starvation. This behavior is neglected in the present but can be considered in future work by applying, for example, the crystal plasticity theory [34] for very thin coating at the (sub)micron scale.

Load control is used for the normal loading until reaching the desired normal load $P$. The initial interference $\omega_{0}$ and the diameter of contact area $d_{0}$ are obtained (see Fig. 1a) when normal loading is completed. Then the displacement-controlled tangential loading $u_{x}$ with increment size $0.01 \omega_{0}$ is applied. The instantaneous tangential stiffness is calculated by Eq. (1) and sliding inception is assumed when this stiffness reduces to $10 \%$ or less of its initial value, which is captured by using a small initial step of $\left(u_{x}\right)_{1}=0.001 \omega_{0}$. The static friction coefficient $\mu$ is determined as $Q_{\max } / P$ (see the theoretical background). The instantaneous contact area $A_{i}$ at each increment of tangential loading is also obtained. Similar to the experimental observation for homogeneous spherical contact in [25], the contact area $A_{i}$ increases as the tangential loading increases. It finally reaches its maximum value $A_{\mathrm{s}}$ (see the diameter $d_{\mathrm{s}}$ in Fig. $1 \mathrm{~b}$ ) at sliding inception. It should be noticed here that an opposite behavior of contact area reduction under tangential loading was observed for elastomers in Ref. [35].

\section{Result and discussion}

By fixing the elastic modulus and the radius of the substrate, i.e., $E_{\mathrm{su}}=200 \mathrm{GPa}$ and $R=10 \mathrm{~mm}$, the model can be determined by four dimensionless parameters: $E_{\mathrm{su}} / Y_{\mathrm{su}} E_{\mathrm{co}} / E_{\mathrm{su}}$ $E_{\mathrm{co}} / Y_{\mathrm{co}}$ and $t / R$. It was shown in [36-38] that the dimensionless tribological parameters, such as the critical load and interference, are only related to the above four dimensionless parameters even for different $E_{\mathrm{su}}$ and $R$ values. The present dimensionless coating thickness $t / R$ differs from previously used normalization by the radius of contact area e.g.
[39]. This provides a more practical way of selecting coating thickness when the radius of the substrate $R$ is known a priori. The radius of the contact area depends on $t$ and load $P$ and hence, is unknown before $t$ and $P$ are selected. This is true also for purely elastic coated spheres. Moreover, the practicality and validity of the dimensionless parameter $t / R$ was demonstrated experimentally in [40].

In the following, the effects of these four dimensionless parameters and the dimensionless normal loading $P^{*}=P / L_{c_{-} \text {co, }}$ within the ranges shown in Table 1 , on $\mu$ are discussed.

The ratio $E_{\mathrm{co}} / Y_{\mathrm{co}}$ covers a wide range from 500 to 1500 since typical values of $E_{\mathrm{co}} / Y_{\text {co }}$ for metals are nearly 1000 as indicated in Ref. [41]. In $P^{*}$ the critical load of the soft coating $L_{c_{-} \text {co }}$ is obtained from Eq. (3). Using the critical load of the coating rather than that of the substrate to normalize the load $P$ is due to the significant contribution of the soft coating to sliding inception over the entire range of coating thicknesses, as will be demonstrated in the next Sect. 4.1.

For the parametric study over the entire range of $t / R$, each of the other parameters in Table 1 varies while all the rest are maintained at their reference values. The range of $t / R$ contains 13 values, including: 0.0005 , six values between 0.001 and 0.006 with a uniform increment 0.001 and six values between 0.008 and 0.03 with variable increments as shown in Table 1. The lower limit of $t / R=0.0005$ is imposed because at lower $t / R$ the elements in the soft coating are excessively distorted by the tangential loading due to very high shear strain. This excessive mesh distortion prevents convergence of the FE method for $t / R<0.0005$ but this problem was unnoticed in [31].

For soft coating materials, the yield strength is less than that of the substrate $\left(Y_{\mathrm{co}}<Y_{\mathrm{su}}\right)$. Thus, the ratio $Y_{\mathrm{co}} / Y_{\mathrm{su}}$ should be less than one. From Table 1 this ratio can be obtained by:

$Y_{\mathrm{co}} / Y_{\mathrm{su}}=\left(E_{\mathrm{co}} / E_{\mathrm{su}}\right) \cdot\left(E_{\mathrm{su}} / Y_{\mathrm{su}}\right) \cdot\left(E_{\mathrm{co}} / Y_{\mathrm{co}}\right)^{-1}$

It turns out that among the $3 \times 3 \times 3=27$ combinations of material properties in Table 1, five combinations resulted in $Y_{\mathrm{co}} / Y_{\mathrm{su}} \geq 1$ and therefore are eliminated from the total number of combinations to be studied. This leaves us with $(3 \times 3 \times 3-5) \times 3=66$ combinations of material properties and normal loads. For each of these combinations, 13

Table 1 Values of the dimensionless parameters

\begin{tabular}{llll}
\hline & Minimum & Reference value & Maximum \\
\hline$E_{\mathrm{co}} / E_{\mathrm{su}}$ & 0.3 & 0.5 & 0.9 \\
$E_{\mathrm{co}} / Y_{\mathrm{co}}$ & 500 & 1000 & 1500 \\
$E_{\mathrm{su}} / Y_{\mathrm{su}}$ & 500 & 1000 & 1500 \\
$P^{*}$ & 50 & 100 & 200 \\
$t / R$ & $0.0005,0.001-0.006,0.008,0.01,0.015,0.02,0.025,0.03$ \\
\hline
\end{tabular}


values of $t / R$ are used hence, the total number of combinations for the soft coating case is $66 \times 13=858$.

\subsection{Effect of dimensionless coating thickness $t / R$}

The static friction coefficient $\mu$ versus the dimensionless coating thickness $t / R$ for the reference case in Table 1 was already presented in [31] (see Fig. 2). However, no detailed physical explanation of the presented behavior was provided in [31]. This will be discussed and explained in the following.

From Table 1 for the material properties of the reference case $\left(P^{*}=P / L_{c_{-} \text {co }}=100\right)$ we have by Eq. (5) $Y_{\text {co }} / Y_{\text {su }}=0.5$. From Eq. (3), since the ratio $E / Y$ is identical for both substrate and coating, the ratio of $L_{c_{\text {s su }}} / L_{c_{\text {c co }}}$ is proportional to $Y_{\text {su }} / Y_{\text {co }}$ and hence, $L_{c_{\_} s u}=2 L_{c_{-} \text {co }}$ (because $\left.Y_{\text {su }} / Y_{\text {co }}=2\right)$. Consequently, under a normal load $P$, we have for the substrate $P / L_{c_{\_} s u}=50$, which shows that the reference load $P^{*}$ causes elastic-plastic deformation in both the coating and substrate materials. The location of plastic deformation depends on material parameters, normal load and coating thickness. In general, for given materials and normal load at very small $t / R$ the accumulated plasticity causing the loss of tangential stiffness mainly occurs in the substrate. Then, as $t / R$ gradually increases, the accumulated plasticity location moves to within the soft coating. For given load and coating thickness, softer coating results in plastic deformation within the coating. For given material and coating thickness, at increasing loads the location of plastic deformation starts in the soft coating and continues into the harder substrate.

In Fig. 2, the static friction coefficient for the minimum coating thickness $t / R=0.0005$ is represented by the value $\mu_{1}$ (that was not shown specifically in [31]). It can be seen that $\mu$ does not approach $\mu_{\mathrm{su}}$ when $t / R$ approaches zero. The sharp drop of $\mu$ from $\mu_{\mathrm{su}}$ at $t / R=0$ to $\mu_{1}$ at $t / R=0.0005$ is clearly seen in Fig. 2. To explain this unique behavior of soft coating, Fig. 4 presents, for soft and hard coatings, a comparison of their relative contributions $\left(u_{x}-u_{s u}\right) / u_{x}$ to the total tangential displacement during the tangential loading process for the case of $t / R=0.0005$. As shown in Fig. 4 , $u_{x}$ is the total tangential displacement at the tip of the spherical contact and $u_{\mathrm{su}}$ is the tangential displacement of the highest point of the substrate. Thus, $\left(u_{x}-u_{\text {su }}\right) / u_{x}$ represents the net contribution of the coating to the total tangential displacement $u_{x}$. For the comparison, the reference case in Table 1, corresponding to soft coating with $Y_{\text {co }} / Y_{\text {su }}=0.5$ was used, and for a hard coating the same reference case but with $E_{\mathrm{co}} / E_{\mathrm{su}}=2$, which by Eq. (5) yields $Y_{\mathrm{co}} / Y_{\mathrm{su}}=2$ was used. The solid dots at the end of the two curves in Fig. 4 correspond to sliding inception. It can be seen that the higher yield strength hard coating presents a significantly low relative contribution to the total

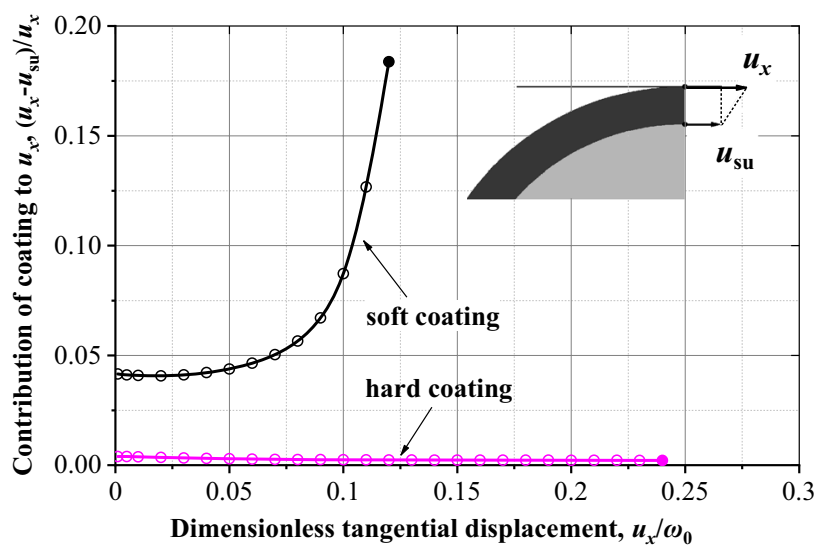

Fig. 4 The relative contributions of soft and hard coatings to tangential displacement during the tangential loading for $t / R=0.0005$ (the solid dots at the end of curves indicate sliding inception)

tangential displacement (less than $0.22 \%$ over the entire range of tangential displacement). It means that in a hard coating case when the coating is very thin $(t / R=0.0005)$, the sliding inception is mainly due to the accumulated plasticity in the softer substrate (which contributes over $99 \%$ of the total tangential displacement). This is the reason why in a hard coating case [30], $\mu$ approaches $\mu_{\text {su }}$ when $t / R$ approaches zero. In the soft coating case, at the early tangential loading stage the relative contribution of the soft coating is already about $5 \%$ and it increases sharply to $18 \%$ at sliding inception. This shows that the soft coating contributes significantly to the total tangential displacement even when it is extremely thin $(t / R=0.0005)$. This may explain why for very thin soft coating $\mu$ does not approach $\mu_{\text {su }}$ but drops sharply once a very thin soft coating is applied. The very sharp drop in friction coefficient shown in Fig. 2 is qualitatively in line with the experimental observation in Ref. [6] (Section 1, Chapter 7) that even the thinnest films of surface contamination, such as oxide layers, adsorbed gases or other contaminants, vastly reduce the friction. Another observation in Fig. 2 (and the experimental study in Ref. [6]), which was not explained in [31], is the presence of minimum friction coefficient at $t / R=(t / R)_{\mathrm{m}}$.

To explain this transitional behavior in Fig. 2, the ratios $\left(u_{s u} / u_{x}\right)_{s}$ and $A_{s} /\left(A_{c o}\right)_{s}$ at sliding inception versus $t / R$ are presented in Fig. 5. Here, $\left(A_{c o}\right)_{s}$ is the contact area at sliding inception of a homogeneous sphere made of the coating material, hence, $\left(A_{c o}\right)_{s}$ is independent of $t / R$. As shown in Fig. 5 , the vertical dashed line at $(t / R)_{\mathrm{m}}$ divides the figure into two regions indicated as I and II. Increasing the dimensionless coating thickness in region I reduces significantly the relative contribution of the substrate from 87 to $45 \%$ of the total tangential displacement. Hence, the soft coating is becoming more and more dominant, enabling higher plasticity level to be accumulated in the contact junction. On 


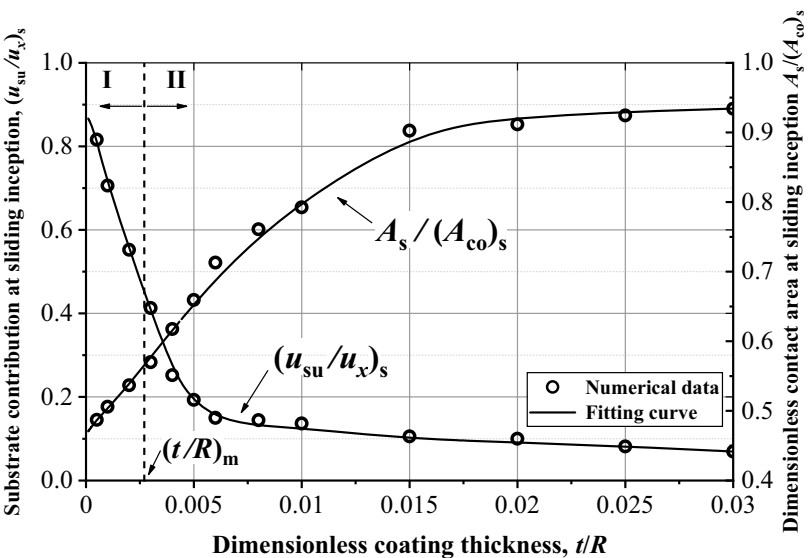

Fig. 5 The relative contribution of substrate and dimensionless contact area at sliding inception as functions of $t / R$ for the reference case in Table 1. The numerical data are fitted by B-spline curve

the other hand, the contact area in region I increases with increasing $t / R$ and hence, for a given normal load $P$, the contact pressure decreases, the plasticity level decreases and offsets the increasing dominancy of the soft coating. As indicated in Sect. 2, static friction coefficient at sliding inception is related to accumulated plasticity and loss of tangential stiffness in the contact junction. It seems that in region I the effect of the soft coating on increasing plasticity level and therefore decreasing tangential stiffness and friction coefficient $\mu$ overcomes the opposite effect of increasing contact area. In region II, the effect of increasing contact area overcomes that of the soft coating growing dominancy and therefore, the plasticity level decreases causing an increase in the tangential stiffness and $\mu$.

A further increase of $t / R$ in region II results in much smaller changes in $\left(u_{\mathrm{su}} / u_{x}\right)_{s}$ and the dimensionless contact area $A_{s} /\left(A_{c o}\right)_{s}$. This explains the asymptotic approach of $\mu$ towards $\mu_{\text {co }}$ at large $t / R$ in Fig. 2.

\subsection{Effects of material properties $E_{\text {su }} / Y_{\text {su' }}, E_{c o} / E_{\text {su' }}$ $E_{\text {co }} / Y_{\text {co }}$}

The effect of $P^{*}$ on $\mu$ was already presented in Fig. 4 of Ref. [31] for $P^{*}=50,100$ and 200, showing that $\mu$ decreases with increasing normal loading for the entire range of $t / R$. Hence, in the following we will focus on the effect of material properties only.

Figure 6a presents the effect of the material parameter $E_{\mathrm{su}} / Y_{\mathrm{su}}$ on $\mu$, showing that a lower $E_{\mathrm{su}} / Y_{\text {su }}$ decreases $\mu$. This can be explained by Fig. $6 \mathrm{~b}$, where the effect of $E_{\mathrm{su}} / Y_{\mathrm{su}}$ on the dimensionless contact area at sliding inception, $A_{s} /$ $\left(A_{\mathrm{co}}\right)_{s}$, is presented. At small $t / R$, a lower $E_{\mathrm{su}} / Y_{\mathrm{su}}$ leads to lower contact area and hence, higher mean contact pressure with more accumulated plasticity in the contact junction that reduces $\mu$. With increasing $t / R$, the effect of $E_{\text {su }} / Y_{\text {su }}$

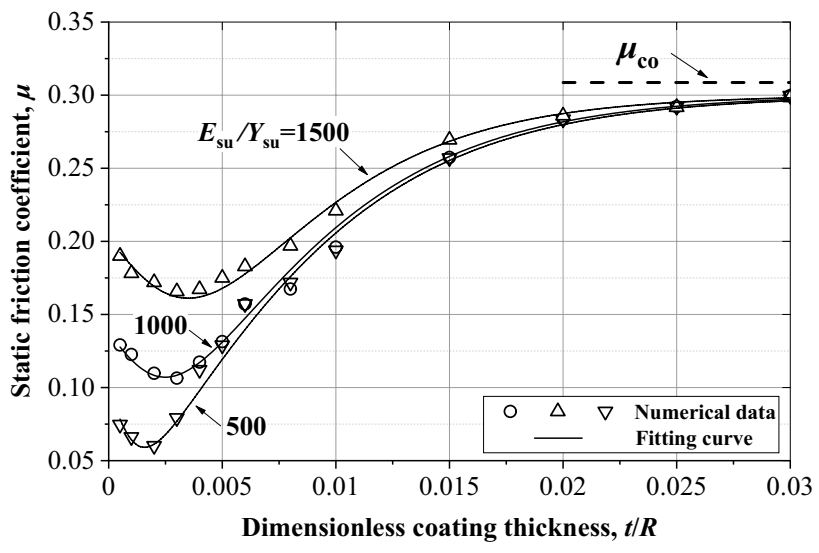

(a)

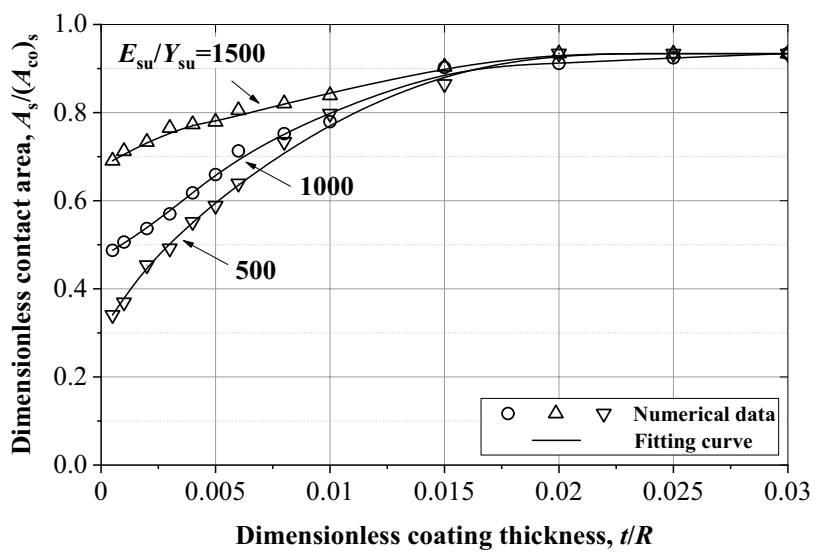

(b)

Fig. 6 The effect of $E_{\mathrm{su}} / Y_{\mathrm{su}}$ on the static friction coefficient $\mu(\mathbf{a})$ and the dimensionless contact area at sliding inception $A_{\mathrm{s}} /\left(A_{\mathrm{co}}\right)_{\mathrm{s}}(\mathbf{b})$ when $E_{\mathrm{co}} / E_{\mathrm{su}}=0.5, E_{\mathrm{co}} / Y_{\mathrm{co}}=1000, P^{*}=100$. The numerical data are fitted by B-spline curve

on the contact area and $\mu$ decreases and $\mu$ approaches $\mu_{\mathrm{co}}$ as would be expected.

Figure 7a presents the effect of $E_{\mathrm{co}} / E_{\mathrm{su}}$ on $\mu$, showing that higher $E_{\mathrm{co}} / E_{\mathrm{su}}$ increases $\mu$. This can be explained by Fig. 7b, where the effect of $E_{\mathrm{co}} / E_{\mathrm{su}}$ on $\left(u_{x}-u_{\mathrm{su}}\right) / u_{x}$ is shown. A higher $E_{\mathrm{co}} / E_{\mathrm{su}}$ corresponds to a lower $\left(u_{x}-u_{\mathrm{su}}\right) / u_{\mathrm{x}}$ for the entire range of $t / R$. As discussed in Sect. 4.1, lower contribution of the soft coating to the tangential displacement leads to a higher $\mu$.

Figure 8a presents the effect of the material parameter $E_{\mathrm{co}} / Y_{\mathrm{co}}$ on $\mu$, showing that a higher $E_{\mathrm{co}} / Y_{\mathrm{co}}$ decreases $\mu$. The reason for this is shown in Fig. $8 \mathrm{~b}$ where a higher $E_{\mathrm{co}} / Y_{\text {co }}$ corresponds to a higher $\left(u_{x}-u_{\mathrm{su}}\right) / u_{x}$ for the entire range of $t / R$, which leads to lower $\mu$.

Note that in Fig. $8 \mathrm{a}, \mathrm{b}$, the lowest value of $E_{\mathrm{co}} / Y_{\mathrm{co}}=800$ is used instead of $E_{\mathrm{CO}} / Y_{\mathrm{CO}}=500$ as in Table 1. This is because the combination of $E_{\mathrm{co}} / Y_{\mathrm{CO}}=500$ with the other two reference values, $E_{\mathrm{co}} / E_{\mathrm{su}}=0.5$ and $E_{\mathrm{su}} / Y_{\mathrm{su}}=1000$, leads according to Eq. (5) to $Y_{\mathrm{co}} / Y_{\mathrm{su}}=1$, which is one of the five eliminated combinations in the present study. 


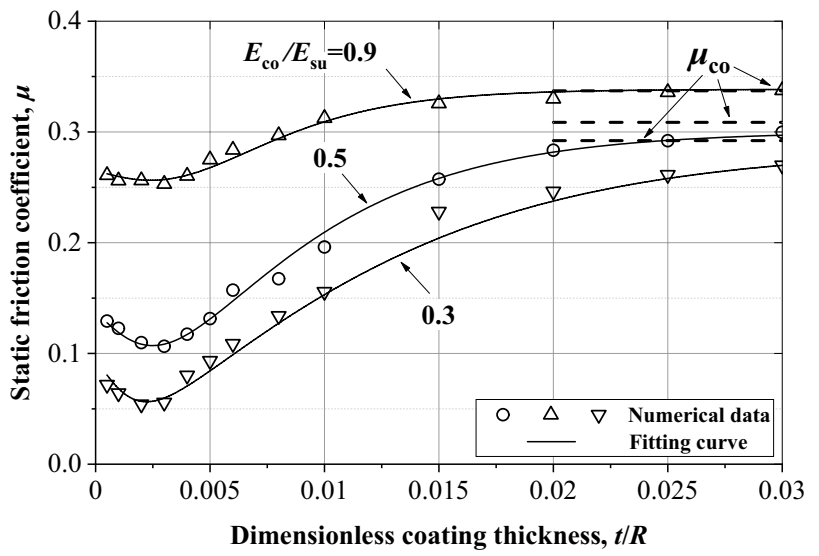

(a)

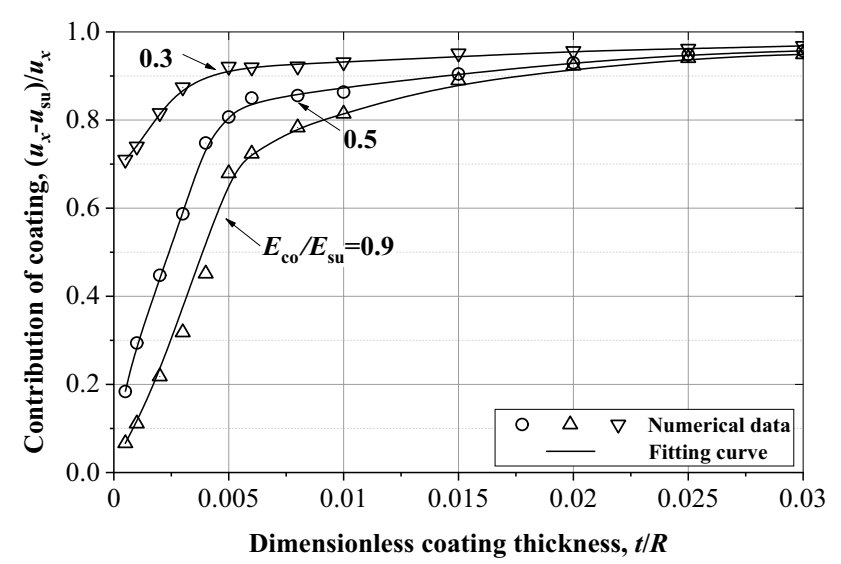

(b)

Fig. 7 The effect of $E_{\mathrm{co}} / E_{\mathrm{su}}$ on static friction coefficient $\mu$ (a) and the contribution of coating to the total tangential displacement $\left(u_{x}-u_{\mathrm{su}}\right) / u_{x}(\mathbf{b})$ when $E_{\mathrm{su}} / Y_{\mathrm{su}}=1000, E_{\mathrm{co}} / Y_{\mathrm{co}}=1000, P^{*}=100$. The numerical data are fitted by $\mathrm{B}$-spline curve

From Figs. 6a, 7a and $8 \mathrm{a}$ one can also see the effect of the various material properties on the amount of initial sharp drop of $\mu$ that was explained in Sect. 4.1. This sharp drop increases for lower $E_{\mathrm{co}} / E_{\mathrm{su}}$ lower $E_{\mathrm{su}} / Y_{\mathrm{su}}$ and higher $E_{\mathrm{co}} / Y_{\mathrm{co}}$, which according to Eq. (5) means lower $Y_{\mathrm{co}} / Y_{\mathrm{su}}$. Hence, the softer the coating is the larger is the initial sharp drop of $\mu$ as would be expected.

It can also be seen from Figs. 6a, 7a and $8 \mathrm{a}$ that $(t / R)_{\mathrm{m}}$ is only slightly affected by $E_{\mathrm{su}} / Y_{\mathrm{su}}$ and negligibly affected by $E_{\mathrm{co}} / E_{\mathrm{su}}$ and $E_{\mathrm{co}} / Y_{\mathrm{co}}$.

\subsection{Expression of $\mu$ for spherical contact with soft coating}

Extensive numerical simulations were performed with all the $66 \times 13=858$ valid combinations in Table 1 , which are relevant to $Y_{\mathrm{co}} / Y_{\mathrm{su}}<1$, over the range of the various

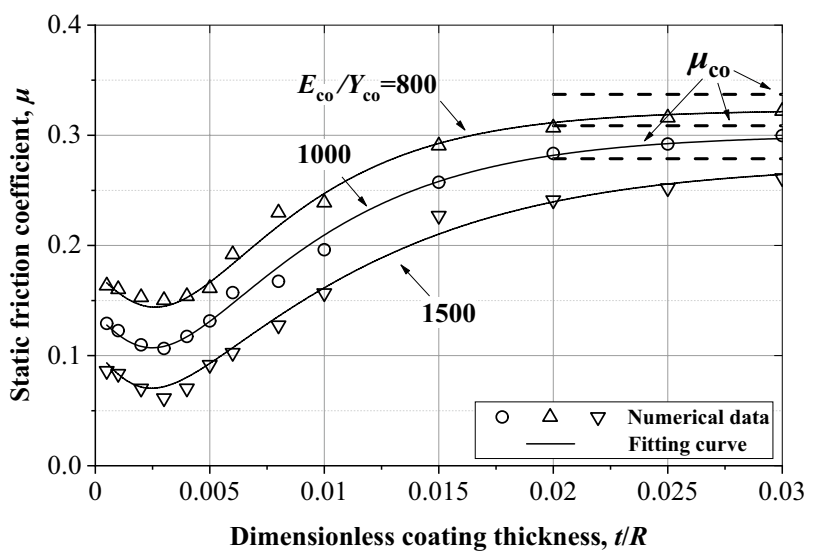

(a)

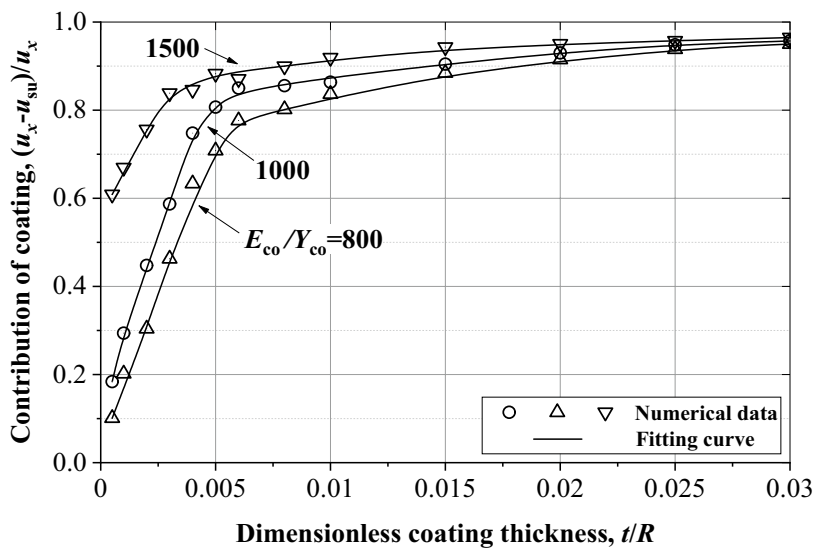

(b)

Fig. 8 The effect of $E_{\mathrm{co}} / Y_{\mathrm{co}}$ on static friction coefficient $\mu$ (a) and the contribution of coating to the total tangential displacement $\left(u_{x}-u_{\mathrm{su}}\right) / u_{x}$ (b) when $E_{\mathrm{co}} / E_{\mathrm{su}}=0.5, E_{\mathrm{su}} / Y_{\mathrm{su}}=1000, P^{*}=100$. The numerical data are fitted by $B$-spline curve

dimensionless parameters. For each of the 66 combinations, the 13 numerical data points from different $t / R$ (see Fig. 2 for example) are fitted by combining two hyperbolic tangent functions in the form:

$\mu=\mu_{1}(1-\tanh (a(t / R-0.0005)))+\mu_{\mathrm{co}} \tanh (b(t / R-0.0005))$

where $\mu_{1}$ is the value of $\mu$ at $t / R=0.0005, a$ and $b$ are fitting parameters and their values for best fit along with the associated standard errors are determined by the "NonlinearModelFit" function in Mathematica (see Ref. [42]). The value of $\mu_{\text {co }}$ is obtained from Eqs. (2) and (3) using the material properties of the coating. With known $\mu_{1}, a, b$, and $\mu_{\mathrm{co}}$ Eq. (6) becomes a function of the form $\mu=\mu(t / R)$. The values of $(t / R)_{\mathrm{m}}$ and $\mu_{\mathrm{m}}$ for each of the 66 combinations are obtained by solving the minimum value of $\mu(t / R)$ (setting its first derivative to zero). Then, globally fitting all the 66 values of $(t / R)_{\mathrm{m}}$ and $\mu_{\mathrm{m}}$ gives their empirical expressions in the form: 
$(t / R)_{\mathrm{m}}=3.65 \times 10^{-6}\left(P^{*}\right)^{0.43}\left(E_{\mathrm{su}} / Y_{\mathrm{su}}\right)^{0.65}$

$\mu_{\mathrm{m}}=13.6\left(P^{*}\right)^{-0.16}\left(E_{\mathrm{co}} / E_{\mathrm{su}}\right)^{1.33}\left(E_{\mathrm{su}} / Y_{\mathrm{su}}\right)^{0.92}\left(E_{\mathrm{co}} / Y_{\mathrm{co}}\right)^{-1.37}$

The goodness of fit for Eq. (7) is $R^{2}=0.997$ and the standard errors of the three fitting parameters on the right hand side are $\delta=0.49 \times 10^{-6}, 0.047$ and 0.066 , respectively. The goodness of fit for Eq. (8) is $R^{2}=0.996$ and the standard errors of the five fitting parameters are $\delta=1.9,0.014,0.024$, 0.026 and 0.025 , respectively. Equation (7) shows that $(t / R)_{\mathrm{m}}$ increases with increasing $P^{*}$ and $E_{\mathrm{su}} / Y_{\mathrm{su}}$. Equation (8) shows that $\mu_{\mathrm{m}}$ decreases for higher $P^{*}$, and for lower $E_{\mathrm{co}} / E_{\mathrm{su}}$ lower $E_{\mathrm{su}} / Y_{\mathrm{su}}$ and higher $E_{\mathrm{co}} / Y_{\mathrm{co}}$, which according to Eq. (5) means lower $Y_{\mathrm{co}} / Y_{\mathrm{su}}$. Hence, the higher the normal load is and the softer the coating is the smaller is $\mu_{\mathrm{m}}$.

By globally fitting all the 66 values of $\mu_{1}$ and $b$, their empirical expressions are given by:

$\mu_{1}=3.6\left(P^{*}\right)^{-0.12}\left(E_{\mathrm{co}} / E_{\mathrm{su}}\right)^{1.01}\left(E_{\mathrm{su}} / Y_{\mathrm{su}}\right)^{0.69}\left(E_{\mathrm{co}} / Y_{\mathrm{co}}\right)^{-0.99}$

$b=13.5\left(P^{*}\right)^{-0.12}\left(E_{\mathrm{co}} / E_{\mathrm{su}}\right)^{0.15}\left(E_{\mathrm{co}} / Y_{\mathrm{co}}\right)^{0.53}-0.19\left(E_{\mathrm{co}} / Y_{\mathrm{co}}\right)$

The goodness of fit for Eq. (9) is $R^{2}=0.994$ and the standard errors of the five fitting parameters on the right hand side are $\delta=0.72,0.017,0.026,0.028$ and 0.027 , respectively. The goodness of fit for Eq. (10) is 0.951 and the standard errors of the five fitting parameters are $\delta=5.9,0.064,0.079,0.22$ and 0.09 , respectively. As can be seen from Eqs. (8) and (9) both $\mu_{\mathrm{m}}$ and $\mu_{1}$ are similarly affected by $P^{*}$ and $Y_{\text {co }} / Y_{\text {su. }}$.

By substituting $(t / R)_{m}, \mu_{m}, \mu_{1}$ and $b$ from Eqs. (7)-(10), respectively, into Eq. (6) the last fitting parameter $a$ is obtained in the form: current metallic materials are considered since in the two different cases the contact area is differently affected by tangential loading, see Ref. [35].

\section{Conclusion}

A soft-coated spherical contact under combined normal and tangential loading was studied using the finite element method. Sliding inception was assumed when the tangential stiffness of the contact junction decreases to $10 \%$ or less of its initial value.

A parametric study was performed, for the first time, to reveal and explain the effects of the dimensionless coating thickness and material properties of coating and substrate on the static friction coefficient.

The initial sharp drop of the static friction coefficient, once a soft coating is applied, and the presence of minimum friction as the dimensionless coating thickness $t / R$ increases (which was previously observed experimentally and theoretically, e.g., $[6,31])$ are due to the competing effects of the soft coating relative contribution to tangential stiffness and of the contact area at sliding inception.

The main conclusions regarding the effect of material properties are: The lower is $Y_{\mathrm{co}} / Y_{\mathrm{su}}$ the larger is the initial sharp drop of static friction coefficient. The optimum dimensionless coating thickness $(t / R)_{\mathrm{m}}$ for minimum friction depends on $E_{\mathrm{su}} / Y_{\mathrm{su}}$ only and increases with increasing this parameter and $P^{*}$. The minimum static friction coefficient $\mu_{\mathrm{m}}$ decreases for softer coating (lower $Y_{\mathrm{co}} / Y_{\text {su }}$ ).

Based on the typical behavior of $\mu$ as a function of $t / R$, an empirical expression for $\mu$ was derived for $t / R \geq 0.0005$.

$a=\frac{1}{(t / R)_{\mathrm{m}}-0.0005} \tanh ^{-1}\left(1-\frac{\mu_{\mathrm{m}}-\mu_{\mathrm{co}} \tanh \left(b\left((t / R)_{\mathrm{m}}-0.0005\right)\right)}{\mu_{1}}\right)$

Hence, an empirical expression for the static friction coefficient $\mu$ for a spherical contact with soft coating can be obtained by substituting Eqs. (7)-(11) in Eq. (6). Therefore, the effects of the dimensionless parameters on $\mu$ can be concluded from their effects on $\mu_{1}$ and $\mu_{\mathrm{co}}$. Under given $P^{*}$ and fixed $\mu_{\text {co }}$ (see Eq. 2), lower $Y_{\text {co }} / Y_{\text {su }}$ (resulting in lower $\mu_{1}$ ) reduce $\mu$. Under given material properties, higher $P^{*}$, which results in both lower $\mu_{1}$ (see Eq. 9) and $\mu_{\text {co }}$ (see Eqs. 2 and 3 ), reduces $\mu$.

It should be noticed here that different results could exist when the mechanism controlling the fate of the junction is that of fracture at the bottom of the spherical asperity instead of the present mechanism dominated by plastic deformation near the contact interface, see Ref. [27]. The results may also be different if elastomer instead of the
It should be noted here that in the present study, the values of $\mu$ for $t / R<0.0005$ are missing due to excessive distortion of the coating elements under tangential loading. To overcome this problem a different method than the current one may be required. Also, a different approach considering fracture mechanism like this in Ref. [27] may be attempted. However, these different methods are out of the scope of the present study and can be attempted in future works.

Acknowledgements This paper is part of IEA AMT IA technical activities. 


\section{Compliance with ethical standards}

Conflict of interest The authors declare that they have no competing interests.

\section{References}

1. Hellgren N (1999) Sputtered carbon nitride thin films. Linköping University, Linköping

2. Holmberg K (1992) A concept for friction mechanisms of coated surfaces. Surf Coat Technol 56(1):1-10

3. Donnet C, Erdemir A (2007) Tribology of diamond-like carbon films: fundamentals and applications. Springer, Berlin

4. Chen L, Liu K, Wei X, Lu Z, Ren N, Zhang G, Xue Q (2019) Enhancement in the tribological properties of CR/DLC multilayers in methane: structural transformation induced by sliding. SN Appl Sci. https://doi.org/10.1007/s42452-019-1521-1

5. Holmberg K, Ronkainen $\mathrm{H}$, Laukkanen A, Wallin K (2007) Friction and wear of coated surfaces - scales, modelling and simulation of tribomechanisms. Surf Coat Technol 202(4-7):1034-1049

6. Bowden FP, Tabor D (1954) The friction and lubrication of solids, vol 1. Oxford University Press, Oxford

7. Finkin $E$ (1969) A theory for the effects of film thickness and normal load in the friction of thin films. J Lubr Technol 91(3):551-555

8. Finkin EF (1971) A theory for the friction of sulfide and other thin films. Wear 18(3):231-241

9. Sadowsky M, Hsu Y, Hussain M (1963) Boundary layers in couplestress elasticity and stiffening of thin layers in shear. Rept.ADWVT-RR-6320. Watervliet Arsenal, Watervliet, New York

10. Rabinowicz E (1967) Variation of friction and wear of solid lubricant films with film thickness. ASLE Trans 10(1):1-9

11. Kato S, Yamaguchi K, Marui E, Tachi K (1982) Frictional properties of a surface covered with a soft metal film-part 2: analysis of friction between a single protuberance and a surface. J Lubr Technol 104(1):39-45

12. Halling $J$ (1979) Surface coatings materials conservation and optimum tribological performance. Tribol Int 12(5):203-208

13. El-Sherbiney M, Halling J (1976) The hertzian contact of surfaces covered with metallic films. Wear 40(3):325-337

14. Chang WR (1997) An elastic-plastic contact model for a rough surface with an ion-plated soft metallic coating. Wear 212(2):229-237

15. Gao F, Kotvis PV, Tysoe WT (2004) The frictional behavior of thin halide films on iron. Tribol Trans 47(2):208-217

16. Liu ZQ, Neville A, Reuben RL (2002) Static friction modeling in the presence of soft thin metallic films. ASME J Tribol 124(1):27-35

17. Liu ZQ, Neville A, Reuben RL (2002) The effect of film thickness on initial friction of elastic-plastically rough surface with a soft thin metallic film. ASME J Tribol 124(3):627-636

18. Derjaguin V, Muller V, Toporov YP (1975) Effect of contact deformations on the adhesion of elastic spheres. J Colloid Inerface Sci 53(3):14-326

19. Valefi M, de Rooij M, Mokhtari M, Schipper DJ (2013) Modelling of a thin soft layer on a self-lubricating ceramic composite. Wear 303(1-2):178-184

20. Mindlin R (1949) Compliance of elastic bodies in contact. ASME J Appl Mech 16:259-268

21. Mindlin R, Deresiewicz H (1953) Elastic spheres in contact under varying oblique forces. ASME J Appl Mech 20:327-344
22. Cattaneo C (1938) Sul contatto di due corpi elastici: Distribuzione locale deglisforzi. Rendiconti dell'Accademia nazionale dei Lincei 27:342-348, 434-436, and 474-478

23. Etsion I (2010) Revisiting the cattaneo-mindlin concept of interfacial slip in tangentially loaded compliant bodies. ASME J Tribol 132(2):020801

24. Brizmer V, Kligerman Y, Etsion I (2007) Elastic-plastic spherical contact under combined normal and tangential loading in full stick. Tribol Lett 25(1):61-70

25. Ovcharenko A, Halperin G, Etsion I (2008) In situ and real-time optical investigation of junction growth in spherical elasticplastic contact. Wear 264(11-12):1043-1050

26. Salib J, Kligerman Y, Etsion I (2008) A model for potential adhesive wear particle at sliding inception of a spherical contact. Tribol Lett 30(3):225-233

27. Aghababaei R, Warner DH, Molinari JF (2016) Critical length scale controls adhesive wear mechanisms. Nat Commun 7:11816

28. Ovcharenko A, Halperin G, Etsion I (2008) Experimental study of adhesive static friction in a spherical elastic-plastic contact. ASME J Tribol 130(2):021401

29. Ghaednia H, Wang X, Saha S, Xu Y, Sharma A, Jackson RL (2017) A review of elastic-plastic contact mechanics. Appl Mech Rev 69(6):060804

30. Chen Z, Etsion I (2018) Model for the static friction coefficient in a full stick elastic-plastic coated spherical contact. Friction 7(6):613-624

31. Chen Z (2019) Friction reduction effect of soft coatings. ASME J Tribol 141(10):104501

32. Brizmer $\mathrm{V}$, Kligerman $\mathrm{Y}$, Etsion I (2007) A model for junction growth of a spherical contact under full stick condition. ASME J Tribol 129(4):783-790

33. Greer JR, Oliver WC, Nix WD (2005) Size dependence of mechanical properties of gold at the micron scale in the absence of strain gradients. Acta Mater 53(6):1821-1830

34. Vakis Al, Yastrebov VA, Scheibert J, Nicola L, Dini D, Minfray $C$, Almqvist A, Paggi M, Lee $S$, Limbert G, Molinari JF, Anciaux G, Aghababaei R, Echeverri Restrepo S, Papangelo A, Cammarata A, Nicolini P, Putignano C, Carbone G, Stupkiewicz S, Lengiewicz J, Costagliola G, Bosia F, Guarino R, Pugno NM, Müser MH, Ciavarella $M$ (2018) Modeling and simulation in tribology across scales: an overview. Tribol Int 125:169-199

35. Sahli R, Pallares G, Ducottet C, Ben Ali IE, Al Akhrass S, Guibert $M$, Scheibert J (2018) Evolution of real contact area under shear and the value of static friction of soft materials. Proc Natl Acad Sci U S A 115(3):471-476

36. Chen Z, Goltsberg R, Etsion I (2016) Plasticity evolution in a coated sphere compressed by a rigid flat. Tribol Int 98:116-124

37. Ronen S, Goltsberg R, Etsion I (2017) A comparison of stick and slip contact conditions for a coated sphere compressed by a rigid flat. Friction 5(3):326-338

38. Chen Z, Goltsberg R, Etsion I (2017) A universal model for a frictionless elastic-plastic coated spherical normal contact with moderate to large coating thicknesses. Tribol Int 114:485-493

39. Komvopoulos K (1988) Finite-element analysis of a layered elastic solid in normal contact with a rigid surface. ASME J Tribol 110(3):477-485

40. Bar-Hen M, Etsion I (2017) Experimental study of the effect of coating thickness and substrate roughness on tool wear during turning. Tribol Int 110:341-347

41. Ashby MF (2005) Materials selection in mechanical design, 3rd edn. Elsevier Butterworth-Heinemann, Oxford

42. Wolfram S (2017) Wolfram mathematica, version 11. Wolfram Research Inc., Champaign

Publisher's Note Springer Nature remains neutral with regard to jurisdictional claims in published maps and institutional affiliations. 